University of South Carolina

Scholar Commons

$5-28-2009$

Bounding Isotropic Lorentz Violation Using Synchrotron Losses at LEP

Brett David Altschul

altschul@mailbox.sc.edu

Follow this and additional works at: https://scholarcommons.sc.edu/phys_facpub

Part of the Physics Commons

Publication Info

Published in Physical Review D, Volume 80, Issue 9, 2009.

(C)2009 American Physical Society

This Article is brought to you by the Physics and Astronomy, Department of at Scholar Commons. It has been accepted for inclusion in Faculty Publications by an authorized administrator of Scholar Commons. For more information, please contact digres@mailbox.sc.edu. 
arXiv:0905.4346

\title{
Bounding Isotropic Lorentz Violation Using Synchrotron Losses at LEP
}

\author{
Brett Altschul] \\ Department of Physics and Astronomy \\ University of South Carolina \\ Columbia, SC 29208
}

\begin{abstract}
Some deviations from special relativity - especially isotropic effects - are most efficiently constrained using particles with velocities very close to 1 . While there are extremely tight bounds on some of the relevant parameters coming from astrophysical observations, many of these rely on our having an accurate understanding of the dynamics of these high-energy sources. It is desirable to have reliable laboratory constraints on these same parameters. The fastest-moving particles in a laboratory were electrons and positrons at LEP. The energetics of the LEP beams were extremely well understood, and measurements of the synchrotron emission rate indicate that the isotropic Lorentz violation coefficient $\left|\tilde{\kappa}_{\mathrm{tr}}-\frac{4}{3} c_{00}\right|$ must be smaller than $5 \times 10^{-15}$.
\end{abstract}

\footnotetext{
${ }^{1}$ baltschu@physics.sc.edu
} 
Presently, there is quite a bit of interest in the possibility that Lorentz and CPT may not be exact symmetries of nature. If the laws of physics, in their most fundamental form at high energies (e.g. at the Planck scale), do not respect these symmetries, then there should be evidence (however weak) of that fact at observable energies. There would be Lorentz- and CPT-violating effects in the effective theory governing low-energy phenomena.

If any violation of Lorentz or CPT invariance were discovered, it would be a breakthrough of profound importance. It would provide crucial information about the structure of fundamental physics and clues as to what other novel effects we could expect to uncover. There is a parameterization of Lorentz and CPT violations in low-energy effective field theory, known as the standard model extension (SME). The SME contains possible Lorentz- and CPT-violating corrections to the standard model [1, 2] and general relativity [3. Both the renormalizability [4, 5] and stability [6] of the SME have been studied.

Historically, there have been a number of phenomenalistic or kinematic frameworks for analyzing the results of Lorentz and CPT tests. However, the SME has now become the standard tool for this purpose. Since it is an effective field theory, it is useful for parameterizing the results of a much broader variety of tests than was possible prior to its inception. Sensitive searches for Lorentz violation have included studies of matter-antimatter asymmetries for trapped charged particles [7, 8, 9] and bound state systems [10, 11], determinations of muon properties [12, 13, analyses of the behavior of spin-polarized matter [14, 15], frequency standard comparisons [16, 17, 18, 19, Michelson-Morley experiments with cryogenic resonators [20, 21], Doppler effect measurements [22, 23], measurements of neutral meson oscillations [24, 25, 26, 27, 28, 29], polarization measurements on the light from cosmological sources [30, 31, 32, 33], high-energy astrophysical tests [34, 35, 36, 37, 38], precision tests of gravity [39, 40, and others. The results of these experiments set bounds on various SME coefficients. Up-to-date information about bounds on the SME coefficients may be found in [41; at the present time, many of the SME coefficients are quite strongly constrained, but many others are not.

One of the most natural ways that Lorentz violation could occur would be to have different sectors of the standard model (e.g. photons and electrons) have different limiting velocities at high energies. However, an isotropic difference in the limiting velocity for two different species turns out to be rather difficult to measure. Direction-dependent effects can be studied by comparing the results of precision experiments performed with the apparatus in different orientations. However, a violation solely of boost invariance requires a comparison of relativistic effects, which are suppressed by two powers of the velocities involved at low energies. The greater the velocities involved in an experiment are, the more precise are the bounds than can be set.

The fastest-moving particles we can study are astrophysical in origin. Astrophysical processes involving electrons and photons have been used to place many strong constraints on SME coefficients. The observed absence of vacuum Cerenkov radiation $\left(e^{-} \rightarrow e^{-}+\gamma\right.$ with superluminal electrons), the absence of photon decay $\left(\gamma \rightarrow e^{+}+e^{-}\right)$, and the structure 
of synchrotron spectra have proved particularly useful. The resulting bounds are typically proportional to $\gamma^{-2}$, where $\gamma$ is the Lorentz factor of the massive particles involved.

However, it is also desirable to have laboratory bounds on the SME coefficients. Many bounds that are based on astrophysical observation rely on our having an accurate understanding of either distant photon sources or high-energy cosmic ray air showers. In most cases, the identities of the particles originally responsible for producing what we actually see can only be inferred, and these inferences may be controversial. For example, there is disagreement whether the $\mathrm{TeV} \gamma$-ray spectra of most sources is caused by inverse Compton scattering or $\pi^{0}$ decay, and whether the highest energy primary cosmic rays are protons, nuclei, or something else entirely. If the particle identifications are incorrect, the corresponding bounds could be completely invalidated. However, a few astrophysical bounds do not suffer from any such such deficiency. In particular, the conclusions drawn from the absence of the process $\gamma \rightarrow e^{+}+e^{-}$do not depend in any way on how the photon involved was produced. The decay process would occur extremely rapidly, if it were allowed. The fact that a photon reaches an Earth-based detector without decaying provides a constraint on Lorentz violation that is completely rigorous, in no way inferior to a measurement with photons both produced and detected in the laboratory.

Vacuum Cerenkov radiation and photon decay are threshold phenomena. In the presence of Lorentz violation, these ordinarily forbidden processes can occur readily. If they are observed not to occur up to an energy $E$, the SME coefficients involved must be smaller than $\mathcal{O}\left(\mathrm{m}^{2} / E^{2}\right)$, where $m$ is the electron mass. Synchrotron radiation is more subtle; it is ordinarily allowed, and Lorentz violation would only result in a change in the radiation spectrum. However, this characteristic can actually be highly advantageous. Precise monitoring of revolving particles' synchrotron losses can be used to constrain the same SME coefficients with significantly better than $\mathcal{O}\left(m^{2} / E^{2}\right)$ precision.

The most highly boosted particles available in a laboratory were electrons and positrons at the Large Electron-Positron Collider (LEP). Energy calibration data from LEP can be used to place very tight constraints on isotropic Lorentz violation. The rate of synchrotron radiation from the electrons and positrons in the accelerator was measured with very high precision, and this fact can be used to better constrain this extremely natural, yet poorly measured in the laboratory, form of Lorentz violation.

The Lagrange density for the electron and photon sectors of the SME is

$$
\begin{aligned}
\mathcal{L} & =-\frac{1}{4} F^{\mu \nu} F_{\mu \nu}-\frac{1}{4} k_{F}^{\mu \nu \rho \sigma} F_{\mu \nu} F_{\rho \sigma}+\frac{1}{2} k_{A F}^{\mu} \epsilon_{\mu \nu \rho \sigma} F^{\nu \rho} A^{\sigma}+\bar{\psi}\left(i \Gamma^{\mu} D_{\mu}-M\right) \psi \\
\Gamma^{\mu} & =\gamma^{\mu}+c^{\nu \mu} \gamma_{\nu}-d^{\nu \mu} \gamma_{\nu} \gamma_{5}+e^{\mu}+i f^{\mu} \gamma_{5}+\frac{1}{2} g^{\lambda \nu \mu} \sigma_{\lambda \nu} \\
M & =m+\not \phi-\not \gamma \gamma_{5}+\frac{1}{2} H^{\mu \nu} \sigma_{\mu \nu}+i m_{5} \gamma_{5} .
\end{aligned}
$$

The behavior of the quanta at high energies is primarily determined by the dimensionless, CPT-even coefficients $c, d$, and $k_{F}$. They affect the velocities of electrons, positrons, and 
photons. However, the $e v^{\mu} A_{\mu}$ coupling between charged particles and the electromagnetic field is not modified; this is a consequence of electromagnetic gauge invariance, which the SME preserves. Since the coefficients parameterizing the Lorentz violation are expected to be small, we shall only consider their leading-order effects.

The LEP energy data is primarily sensitive to isotropic Lorentz violation, given the bounds that have already been placed on the various SME parameters. In the photon sector the nineteen-component, double traceless $k_{F}$ can be separated into coefficients that lead to photon birefringence and those which do not. The former are very strongly constrained by cosmological measurements [31, 32, 33]. Of the coefficients that are not related to vacuum birefringence, the ones which are even under parity are already constrainedusing resonant cavity experiments [20, 21] - at roughly the $10^{-17}$ level; the crucial exception is the isotropic coefficient $\tilde{\kappa}_{\mathrm{tr}}=\frac{2}{3}\left(k_{F}\right)_{\alpha}{ }^{0 \alpha 0}$. The parity-odd ones are less well bounded, but they have little effect on the rate of synchrotron losses at an accelerator. They may affect the instantaneous power emitted, but the total power loss over a full revolution - being a parity-even quantity - is unchanged; any increase in radiation along one side of the orbit is compensated by a decrease on the opposite side. Therefore, of the photon-sector coefficients, only the isotropic boost invariance violation coefficient $\tilde{\kappa}_{\text {tr }}$ can affect the synchrotron power at the level of interest.

In the electron sector, the $c$ coefficients affect the velocities of electrons and positrons. The same resonant cavity experiments used to constrain the non-birefringent $k_{F}$ coefficients can also constrain the $c$ coefficients directly, taking advantage of the dependence of a cavity's shape on the electron-sector parameters. The parity even $c_{j k}$ coefficients are constrained tightly enough to be neglected here, and the parity-odd $c_{0 j}$ do not affect the total power emitted during an orbit. This leaves $c_{00}$, which is relatively poorly constrained by laboratory experiments. However, using a coordinate redefinition, $x^{\prime \mu}=x^{\mu}-c^{\mu}{ }_{\nu} x^{\nu}$ [42], it is actually possible to eliminate $c$ from the Langragian; only differences between the $c$ and non-birefringent $k_{F}$ coefficients are physically measurable. Although we shall use this freedom to set $c^{\nu \mu}=0$ and henceforth only consider $\tilde{\kappa}_{\text {tr }}$, the bounds we shall derive will more generally be on the combination $\tilde{\kappa}_{\mathrm{tr}}-\frac{4}{3} c_{00}$. The best astrophysical bounds on this quantity, disentangled from the other all other coefficients, are $-1.3 \times 10^{-14}<\tilde{\kappa}_{\mathrm{tr}}-\frac{4}{3} c_{00}<8 \times 10^{-15}$ [37]. The laboratory bounds derived here are comparable and more secure.

The $d$ coefficients are analogous to the birefringent part of $k_{F}$. Their effects are similar to those of $c$, except that they depend on helicity and particle-antiparticle identity. The $d$ coefficients only affect synchrotron radiation losses if the beams are longitudinally polarized, which can be the case instantaneously but not over long periods. Electron helicity precesses in a magnetic field, because of the anomalous magnetic moment. For this reason, the LEP beam was ordinarily maintained in a transverse polarization state and only rotated into longitudinal polarization before an interaction point.

It has recently been observed that accelerator data could be used to place new laboratory constraints on $\tilde{\kappa}_{\text {tr }}$ [43]. The absence of vacuum Cerenkov radiation at LEP indicates 
that $\tilde{\kappa}_{\mathrm{tr}}<1.2 \times 10^{-11}$. The fact that energetic photons produced at the Tevatron do not decay shows that $\tilde{\kappa}_{\mathrm{tr}}>-5.8 \times 10^{-12}$. However, it is possible to place much stronger bounds than these, using the same LEP energy data, by taking advantage of the high precision to which the LEP synchrotron losses were determined.

The synchrotron process in the presence of Lorentz violation was discussed in detail in [44]. However, for the case of isotropic Lorentz violation only, the main changes can be understood quite simply. The inclusion of $\tilde{\kappa}_{\mathrm{tr}}$ in the Lagrangian changes the propagation speed of photons to $\sqrt{\frac{1-\tilde{\kappa}_{\mathrm{tr}}}{1+\tilde{\kappa}_{\mathrm{tr}}}} \approx 1-\tilde{\kappa}_{\mathrm{tr}}$. The electromagnetic sector behaves according to ordinary special relativity, except with a modified Lorentz factor $\tilde{\gamma}=\left(1-2 \tilde{\kappa}_{\mathrm{tr}}-v^{2}\right)^{-1 / 2}$. The power radiated by a synchrotron electron is $P=\frac{e^{2} a^{2}}{6 \pi m^{2}} \tilde{\gamma}^{4}$, where $a$ is the magnitude of the acceleration; the electron velocity is effectively increased to $v+\tilde{\kappa}_{\mathrm{tr}}$. (If an electron's velocity exceeds $1-\tilde{\kappa}_{\text {tr }}$, vacuum Cerenkov radiation will be emitted.) For ultrarelativistic particles, $\gamma \approx[2(1-v)]^{-1 / 2}$ is a very sensitive function of $v$, with $d \gamma / d v=v \gamma^{3} \approx \gamma^{3}$. In the presence of the Lorentz violation the radiated power becomes

$$
P=P_{0}\left(1+4 \gamma^{2} \tilde{\kappa}_{\mathrm{tr}}\right),
$$

where $P_{0}$ is the radiation rate in the absence of $\tilde{\kappa}_{\text {tr }}$.

Precise determination of the beam energy at LEP was important, since one of the accelerator's most important functions was to provide precision measurements of the W and $\mathrm{Z}$ boson masses. The beam energy $E$ was calculated using several complementary methods. The first method entailed measuring the magnetic field profile using nuclear magnetic resonance (NMR) and also measuring the beam trajectory; together these determine the beam energy. The field strength and the radius of the orbit in the bending magnets were known to high precision. Moreover, the validity of the NMR measurements would not be affected by Lorentz violation; any Lorentz violations strong enough to affect the magnetic field measurements are ruled out by atomic clock experiments [16, 17, 18, 19].

Also measured was the synchrotron tune, $Q_{s}$ - the ratio of the synchrotron oscillation frequency to the orbital frequency [45]. The oscillations occurred because of the nonuniformity in the beam particles' energies. Particles with less than the nominal beam energy revolve around smaller paths, and thus they travel between the radio frequency (RF) accelerating cavities more quickly. They arrive at the cavities earlier in the RF cycle and receive larger-than-expected energy boosts. The opposite occurs for particle with greater than the nominal energy. This effect causes synchrotron oscillations in the beam, and a fit to their frequency provides an independent way to determine $E$.

The fit of $Q_{s}$ produced a measurement of $E$ with a $1 \sigma$ uncertainty of $21 \mathrm{MeV}$ (on a 91 GeV run) [45]. This uncertainty was much larger than the discrepancy of $3 \mathrm{MeV}$ between the values of the energy inferred from $Q_{s}$ and from NMR. The uncertainty is primarily controlled by the fitting uncertainty and the precision with which $Q_{s}$ is measured. $E$ and $U_{0}$ (the energy loss per revolution) enter the formula for $Q_{s}$ through $\left(g^{2} e^{2} V_{R F}^{2}-U_{0}^{2}\right) / E^{2}$, and under the steady state conditions at which the collider operated, $U_{0}=g e V_{R F} \sin \psi_{s}$. 
$V_{R F}$ and $\psi_{s}$ are the amplitude and phase of the RF voltage during the beam's passage through the accelerating cavities; both are known to high precision. $g$ is a correction factor related to possible phasing errors and misalignments of the cavities. It is a fit parameters and a significant source of uncertainty; however, in absolute terms, its value is close to 1 , and its value can be determined using a separate fit to $Q_{s}$ performed well below real experimental energies. The presence of $\tilde{\kappa}_{\text {tr }}$ does not change the formula for $Q_{s}$, except through a rescaling of $U_{0}$; since the Lorentz force law is not modified, the motion of the particles in the applied fields and the energy imparted by the RF cavities are unchanged.

$U_{0}$ was not a parameter that was varied in the fit; it was assumed to take the conventional synchrotron form, corrected to account for additional well understood losses (primarily related to finite beam size and parasitic impedance interactions; these and other corrections were either modeled from first principles or measured directly). However, since $E$ was independently and more accurately known from NMR measurements, it is possible to reinterpret the fit for $E$ as a fit for $U_{0}$. Since $E$ and $U_{0}$ enter the formula for $Q_{s}$ only in the combination $-\left(U_{0} / E\right)^{2} \cos ^{2} \psi_{s}$, the uncertainty ascribed to $E$ in the fit for the energy is equivalent to essentially the same fractional uncertainty of $2.4 \times 10^{-4}$ in $U_{0}$ (and hence $P$ ).

Conservatively, we may state that the fractional deviation of $P$ from its conventionally expected value is $\eta<6 \times 10^{-4}$, for measurements performed at the $\mathrm{Z}$ pole energy of 91 $\mathrm{GeV}$ (corresponding to $\gamma>1.7 \times 10^{5}$ ). This represents a $2 \sigma$ bound, and it accounts for all additional sources of error, such as the error in the NMR measurement of $E$ and the discrepancy between that measured value and the value inferred from $Q_{s}$. Then according to (4),

$$
\left|\tilde{\kappa}_{\text {tr }}\right|<\frac{\eta}{4 \gamma^{2}}<5 \times 10^{-15} .
$$

This bound is not as strong as the bound on $\tilde{\kappa}_{\text {tr }}$ that comes from the absence of photon decay [34]. The absence of $\gamma \rightarrow e^{+}+e^{-}$for up to $50 \mathrm{TeV}$ photons gives a bound at the $2 \times 10^{-16}$ level, although that bound is strictly one-sided; only negative values of $\tilde{\kappa}_{\text {tr }}$ are so constrained. Moreover, the photon decay bounds are also entangled with bounds on the parity-odd coefficients. Therefore, the current result represents an improvement in clean, reliable, laboratory-derived bounds of three orders of magnitude. This improvement over the vacuum Cerenkov bounds comes precisely from the $\lesssim 10^{-3}$ precision with which the synchrotron loss rate is known.

While the parity-odd coefficients $\tilde{\kappa}_{o+}$ (or equivalently $c_{0 j}$ ) do not contribute to the energy lost during a full revolution, they do affect the instantaneous rate of of synchrotron emission. There is an additional, direction-dependent modification of the speed of light, which, in the presence of a generic non-birefringent $k_{F}$ is $1-\frac{1}{2}\left[\tilde{k}_{j k} \hat{v}_{j} \hat{v}_{k}+2 \tilde{k}_{0 j} \hat{v}_{j}+\tilde{k}_{00}\right]$, where $\tilde{k}^{\mu \nu}=\left(k_{F}\right)_{\alpha}{ }^{\mu \alpha \nu}$. The $\tilde{k}_{0 j}$ are equivalent to $\epsilon_{j k l}\left(\tilde{\kappa}_{o+}\right)^{k l}$ and the $\tilde{k}_{j k}$ to $\left(\tilde{\kappa}_{e-}\right)_{j k}$. These generalize the isotropic case, which has $\tilde{k}_{00}=\frac{3}{2} \tilde{\kappa}_{\text {tr }}$ and $\tilde{k}_{j k}=\frac{1}{2} \tilde{\kappa}_{\text {tr }} \delta_{j k}$. With more detailed data on the emission rates of the LEP electrons and positrons as they moved, one could potentially place bounds (comparable to the $\tilde{\kappa}_{\text {tr }}$ bounds) on the $\tilde{\kappa}_{o+}$ parameters. 
In summary, we have derived new bounds, based on terrestrial laboratory experiments, on the isotropic Lorentz violation parameter $\tilde{\kappa}_{\text {tr }}$. The precision with which synchrotron losses at LEP matched conventional expectations constrains electron and photon SME

parameters to be $\left|\tilde{\kappa}_{\text {tr }}-\frac{4}{3} c_{00}\right|<5 \times 10^{-15}$. This new two-sided constraint represents an improvement of three orders of magnitude over previous laboratory bounds on these quantities. It also represents a modest improvement over the best astrophysical bounds on $\tilde{\kappa}_{\text {tr }}-\frac{4}{3} c_{00}$ alone.

\section{Acknowledgments}

The author is grateful to M. Purohit for helpful discussion.

\section{References}

[1] D. Colladay, V. A. Kostelecký, Phys. Rev. D 55, 6760 (1997).

[2] D. Colladay, V. A. Kostelecký, Phys. Rev. D 58, 116002 (1998).

[3] V. A. Kostelecký, Phys. Rev. D, 69105009 (2004).

[4] V. A. Kostelecký, C. D. Lane, A. G. M. Pickering, Phys. Rev. D 65, 056006 (2002).

[5] D. Colladay, P. McDonald, Phys. Rev. D 75, 105002 (2007).

[6] V. A. Kostelecký, R. Lehnert, Phys. Rev. D 63, 065008 (2001).

[7] R. Bluhm, V. A. Kostelecký, N. Russell, Phys. Rev. Lett. 79, 1432 (1997).

[8] G. Gabrielse, A. Khabbaz, D. S. Hall, C. Heimann, H. Kalinowsky, W. Jhe, Phys. Rev. Lett. 82, 3198 (1999).

[9] H. Dehmelt, R. Mittleman, R. S. Van Dyck, Jr., P. Schwinberg, Phys. Rev. Lett. 83, 4694 (1999).

[10] R. Bluhm, V. A. Kostelecký, N. Russell, Phys. Rev. Lett. 82, 2254 (1999).

[11] D. F. Phillips, M. A. Humphrey, E. M. Mattison, R. E. Stoner, R. F. C. Vessot, R. L. Walsworth , Phys. Rev. D 63, 111101 (R) (2001).

[12] R. Bluhm, V. A. Kostelecký, C. D. Lane, Phys. Rev. Lett. 84, 1098 (2000).

[13] V. W. Hughes, et al., Phys. Rev. Lett. 87, 111804 (2001).

[14] R. Bluhm, V. A. Kostelecký, Phys. Rev. Lett. 84, 1381 (2000). 
[15] B. R. Heckel, C. E. Cramer, T. S. Cook, S. Schlamminger, E. G. Adelberger, U. Schmidt, Phys. Rev. Lett. 97, 021603 (2006).

[16] C. J. Berglund, L. R. Hunter, D. Krause, Jr., E. O. Prigge, M. S. Ronfeldt, S. K. Lamoreaux, Phys. Rev. Lett. 75, 1879 (1995).

[17] V. A. Kostelecký, C. D. Lane, Phys. Rev. D 60, 116010 (1999).

[18] D. Bear, R. E. Stoner, R. L. Walsworth, V. A. Kostelecký, C. D. Lane, Phys. Rev. Lett. 85, 5038 (2000).

[19] P. Wolf, F. Chapelet, S. Bize, A. Clairon, Phys. Rev. Lett. 96, 060801 (2006).

[20] H. Müller, et al., Phys. Rev. Lett. 99, 050401 (2007).

[21] S. Herrmann, A. Senger, K. Möhle, E. V. Kovalchuk, A. Peters, in CPT and Lorentz Symmetry IV, edited by V. A. Kostelecký (World Scientific, Singapore, 2008), p. 9.

[22] G. Saathoff, S. Karpuk, U. Eisenbarth, G. Huber, S. Krohn, R. Muñoz Horta, S. Reinhardt, D. Schwalm, A. Wolf, G. Gwinner, Phys. Rev. Lett. 91, 190403 (2003).

[23] C. D. Lane, Phys. Rev. D 72, 016005 (2005).

[24] V. A. Kostelecký, Phys. Rev. Lett. 80, 1818 (1998).

[25] V. A. Kostelecký, Phys. Rev. D 61, 016002 (1999).

[26] Y. B. Hsiung, Nucl. Phys. Proc. Suppl. 86, 312 (2000).

[27] K. Abe et al., Phys. Rev. Lett. 86, 3228 (2001).

[28] J. M. Link et al., Phys. Lett. B 556, 7 (2003).

[29] B. Aubert et al., Phys. Rev. Lett. 96, 251802 (2006).

[30] S. M. Carroll, G. B. Field, Phys. Rev. Lett. 79, 2394 (1997).

[31] V. A. Kostelecký, M. Mewes, Phys. Rev. Lett. 87, 251304 (2001).

[32] V. A. Kostelecký, M. Mewes, Phys. Rev. Lett. 97, 140401 (2006).

[33] V. A. Kostelecký, M. Mewes, Phys. Rev. Lett. 99, 011601 (2007).

[34] F. W. Stecker, S. L. Glashow, Astropart. Phys. 16, 97 (2001).

[35] T. Jacobson, S. Liberati, D. Mattingly, Nature 424, 1019 (2003).

[36] B. Altschul, Phys. Rev. Lett. 96, 201101 (2006). 
[37] B. Altschul, Phys. Rev. D 74, 083003 (2006).

[38] F. R. Klinkhamer, M. Risse, Phys. Rev. D 77, 016002 (2008); addendum Phys. Rev. D 77, 117901 (2008).

[39] J. B. R. Battat, J. F. Chandler, C. W. Stubbs, Phys. Rev. Lett. 99, 241103 (2007).

[40] H. Müller, S. Chiow, S. Herrmann, S. Chu, Phys. Rev. Lett. 100, 031101 (2008).

[41] V. A. Kostelecký, N. Russell, arXiv:0801.0287.

[42] Q. G. Bailey, V. A. Kostelecký, Phys. Rev. D 70, 076006 (2004).

[43] M. A. Hohensee, R. Lehnert, D. F. Phillips, R. L. Walsworth, Phys. Rev. Lett. 102, $170402(2009)$.

[44] B. Altschul, Phys. Rev. D 72, 085003 (2005).

[45] R. Assmann, et al., Eur. Phys. J. C 39, 253 (2005). 